\title{
Some characterizations of dual vector fields
}

Hatice Kusak Samanci and Serkan Celik

Department of Mathematics, Faculty of Science and Arts, Bitlis Eren University, Bitlis, Turkey

Received: 9 January 2016, Accepted: 6 May 2016

Published online: 25 June 2016.

\begin{abstract}
The set of the dual vectors which are introduced by $\mathbf{A}=\mathbf{a}+\varepsilon \mathbf{a}^{*}, \varepsilon^{2}=0$ called as dual vector field. In our paper, we introduce the directional derivative of the dual vector fields and investigate some properties of them. Then we give a numeric example of the dual vector field aided by E.Study theorem.
\end{abstract}

Keywords: Dual space, directional derivative, dual vector field.

\section{Introduction}

Dual number was first introduced by W.K. Clifford in 1873, [1]. If $a$ and $a^{*}$ are real numbers then the combination, $A=a+\varepsilon a^{*}$ called a dual number and the symbol $\varepsilon$ denotes the dual unit with the property that $\varepsilon^{2}=0$. Application of this were studied by A.P. Kotelnikov in 1895, [1]. Then, E. Study used the dual numbers and dual vectors in his research on the geometry of lines and kinematics and defined the mapping which is called with his name: There is one to one correspondence between an oriented straight line in Euclidean 3-space and a dual point on surface of a dual unit sphere in dual space, [2,3]. Dual vectors in instantaneous spatial kinematics were studied by G.R. Veldkamp, in 1976, [4]. Covariant derivative of a dual vector field in the direction another dual vector field was accomplished the dual space in the reference [5]. Dual vector algebra provides a convenient tool for handling mathematical entities such as screw, [6].

The aim of this paper is to completely define a directional derivative on $\mathrm{n}$ dimensional dual space. So our intention is to apply several new concepts on dual space defined in differential geometry $[7,8]$.

\section{Preliminaries}

A dual number is an expression of the form $A=a+\varepsilon a^{*}$, where $a$ and $a^{*}$ are real numbers and the dual unit $\varepsilon$ satisfied the condition $\varepsilon^{2}=0, \varepsilon \neq 0$. We denote the set of dual numbers by

$$
\mathbb{D}=\left\{A \mid A=a+\varepsilon a^{*}, a, a^{*} \in \mathbb{R}\right\} .
$$

The addition and multiplication of dual numbers are

$$
\begin{gathered}
A+B=(a+b)+\varepsilon\left(a^{*}+b^{*}\right) \\
A . B=(a \cdot b)+\varepsilon\left(a \cdot b^{*}+b \cdot a^{*}\right) \\
\lambda A=\lambda a+\varepsilon \lambda a^{*} .
\end{gathered}
$$


The set $\mathbb{D}$ of dual numbers with the above operations is a commutative ring. The elements of the set

$$
\mathbb{D}^{n}=\left\{\mathbf{A} \mid \mathbf{A}=\mathbf{a}+\varepsilon \mathbf{a}^{*}, \mathbf{a}, \mathbf{a}^{*} \in \mathbb{R}^{n}\right\}
$$

are called $n$-dimensional dual vectors on the $n$-dimensional dual space. An $n$-dimensional dual vector $\mathbf{A}$ maybe expressed in the form $\mathbf{A}=\mathbf{a}+\varepsilon \mathbf{a}^{*}=\left(\mathbf{a}, \mathbf{a}^{*}\right)$ where $\mathbf{a}=\left(a_{1}, a_{2}, \ldots, a_{n}\right)$ and $\mathbf{a}^{*}=\left(b_{1}, b_{2}, \ldots, b_{n}\right)$ are vectors of $\mathbb{R}^{n}$.

Theorem 1. Let $\mathbf{A}, \mathbf{B} \in \mathbb{D}^{n}$ be the $n$-dimensional dual vectors. The addition of two dual vectors are

$$
\mathbf{A}+\mathbf{B}=(\mathbf{a}+\mathbf{b})+\varepsilon\left(\mathbf{a}^{*}+\mathbf{b}^{*}\right)
$$

Theorem 2. When $\mathbf{A}, \mathbf{B} \in \mathbb{D}^{n}$ are n-dimensional dual vectors, inner product of $A, B$ dual vectors are computed with the following formula :

$$
<\mathbf{A}, \mathbf{B}>=<\vec{a}, \vec{b}>+\varepsilon\left(<\overrightarrow{a^{*}} \vec{b}>+<\vec{a}, \vec{b}^{*}>\right)
$$

Theorem 3. $\mathbf{A}=\mathbf{a}+\varepsilon \mathbf{a}^{*} \in \mathbb{D}^{n}$ the norm of a dual vector is computed with:

$$
\|\mathbf{A}\|^{2}=\|\mathbf{a}\|^{2}+2 \varepsilon<\mathbf{a}, \mathbf{a}^{*}>
$$

Definition 1. If $\vec{v}_{p} \in T_{E}^{n}$ and $f \in C\left(\mathbb{E}^{n}, \mathbb{R}\right)$; the derivative of the $f$ function in the direction vector $\vec{v}_{p}$ is defined with: $v_{p}[f]=<\mathbf{v},\left.\overrightarrow{\nabla f}\right|_{P}>$.

\section{Main results}

Theorem 4. $\mathbb{D}^{n}$ space is an affine space,

$$
\begin{aligned}
F: \mathbb{D}^{n} \times \mathbb{D}^{n} & \longrightarrow V \\
(P, Q) & \longrightarrow F(P, Q)=\overrightarrow{P Q}
\end{aligned}
$$

(A1) For $\forall P, Q, R \in \mathbb{D}^{n} F(P, Q)+F(Q, R)=F(P, R)$

(A2) For $\forall P \in \mathbb{D}^{n}, \forall \mathbf{A} \in \mathbb{D}^{n}, \overrightarrow{P Q}=\mathbf{A}$ there is the $\exists Q \in \mathbb{D}^{n}$ properties are observed.

Proof. $\forall P=\mathbf{p}+\varepsilon \mathbf{p}^{*}, Q=\mathbf{q}+\varepsilon \mathbf{q}^{*}$ and $R=\mathbf{r}+\varepsilon \mathbf{r}^{*} \in \mathbb{D}^{n}$ here for $\mathbf{p}, \mathbf{q}, \mathbf{r}, \mathbf{p}^{*}, \mathbf{q}^{*}, \mathbf{r}^{*} \in \mathbb{E}^{n}$ and $A=\mathbf{a}+\varepsilon \mathbf{a}^{*} \in \mathbb{D}$ dual vector, $\mathbf{a}, \mathbf{a}^{*} \in \mathbb{E}^{n}$.

(A1) Let us show that the first of one of the affine axioms is obtained:

$$
\begin{aligned}
F(P, Q) & =\mathbf{P Q}=Q-P=\left(\mathbf{q}+\varepsilon \mathbf{q}^{*}\right)-\left(\mathbf{p}+\varepsilon \mathbf{p}^{*}\right) \\
& =(q-p)+\varepsilon\left(\mathbf{q}^{*}-\mathbf{p}^{*}\right) \\
F(Q, R) & =\mathbf{Q R}=R-Q=\left(\mathbf{r}+\varepsilon \mathbf{r}^{*}\right)-\left(\mathbf{q}+\varepsilon \mathbf{q}^{*}\right) \\
& =(r-p)+\varepsilon\left(\mathbf{r}^{*}-\mathbf{q}^{*}\right)
\end{aligned}
$$

It is observed that by adding the above equations side by side, it is observed that,

$$
F(P, Q)+F(Q, R)=(r-p)+\varepsilon\left(\mathbf{r}^{*}-\mathbf{p}^{*}\right)=F(P, R) .
$$


By so-doing, the first of the affine axioms for $\mathbb{D}^{n}$ space is proven.

(A2) Let us show that the second of the affine axioms is obtained:

$$
\begin{aligned}
(P, Q) \longrightarrow F(P, Q) & =\mathbf{P Q}=Q-P \\
& =\left(\vec{q}+\varepsilon \vec{q}^{*}\right)-\left(\vec{p}+\varepsilon \vec{p}^{*}\right) .
\end{aligned}
$$

If we equalize the $\mathbf{A}=\vec{a}+\varepsilon \vec{a}^{*}$ dual vector and the result obtained in the Equation (1) to each other, we find the $\vec{q}=\vec{a}+\vec{p}$ and $\vec{q}^{*}=\vec{a}^{*}+\vec{p}^{*}$. So, we can say that the following point exists:

$$
Q=\mathbf{q}+\varepsilon \vec{q}^{*}=(\vec{a}+\vec{p})+\varepsilon\left(\vec{a}^{*}+\overrightarrow{p^{*}}\right) \in \mathbb{D}^{n} .
$$

As a consequence, since (A1) and (A2) axioms are obtained, it is observed that the dual space $\mathbb{D}^{n}$ is an affine space.

Definition 2. For the dual point $P \in \mathbb{D}^{n}$, and the dual vector $\vec{A} \in \mathbb{D}^{n},(P, \vec{A})$ in the dual space $\mathbb{D}^{n}$ is called as the dual tangent vector, and shown with $\vec{A}_{p}$.

Definition 3. In $P \in \mathbb{D}^{n}$ point,

$$
T_{\mathbb{D}^{n}}(P)=\vec{A}_{p}=(P, \vec{A}): P \in \mathbb{D}^{n}, A \in \mathbb{D}-\text { modul }
$$

set is called as dual tangent vectors set. Besides, if $c \in \mathbb{R}$ and $\forall \vec{A}_{p}, \vec{B}_{p} \in T_{\mathbb{D}^{n}}(P)$

$$
\begin{aligned}
\vec{A}_{p} \oplus \vec{B}_{p} & =(P, \vec{A})+(P, \vec{B})=(P, \vec{A}+\vec{B})=(\vec{A}+\vec{B})_{p} \\
c \cdot \vec{A}_{p} & =c \cdot(P, \vec{A})=(P, c \cdot \vec{A})
\end{aligned}
$$

$\left\{T_{\mathbb{D}^{n}}(P), \oplus, \mathbb{R},+, ., \odot\right\}$ vector space of the dual affine space $\mathbb{D}^{n}$ for the point $P$ is called as dual tangent vectors space for the point $P$, shortly, dual tangent space.

Definition 4. If $\mathbf{A}_{p} \in T_{\mathbb{D}}^{n}(P)$ dual vector, $F$ differentiable dual function, the derivative of the dual function $F$ in the direction of dual vector $A_{p}$ is given with;

$$
A_{p}[F]=<\mathbf{A}_{p},\left.\overrightarrow{\nabla F}\right|_{p}>
$$

Theorem 5. If dual vector $\mathbf{A}_{p} \in T_{D}^{n}(P)$, the derivation of dual function $F$ in the direction of the dual vector $A_{p}$ may also be shown with the following equation:

$$
A_{p}[F]=<\mathbf{a}, \overrightarrow{\nabla f}>+\varepsilon\left[<a^{*}, \overrightarrow{\nabla f}>+<\overrightarrow{\nabla f}^{*}, \mathbf{a}>\right] .
$$


Proof.Let us take the $F=f\left(x_{1}, x_{2}, x_{3}\right)+\varepsilon f^{*}\left(x_{1}, x_{2}, x_{3}\right)$ dual function for $f, f^{*} \in C\left(\mathbb{E}^{n}, \mathbb{R}\right)$;

$$
\begin{aligned}
A_{p}[F] & =<\mathbf{A}_{p},\left.\overrightarrow{\nabla F}\right|_{p}> \\
& =<\mathbf{a}+\varepsilon \mathbf{a}^{*},\left.\left(\frac{\partial F}{\partial x_{1}}, \frac{\partial F}{\partial x_{2}}, \frac{\partial F}{\partial x_{3}}\right)\right|_{p}> \\
& \left.=<\mathbf{a}+\varepsilon \mathbf{a}^{*}, \frac{\partial f+\varepsilon f^{*}}{\partial x_{1}}, \frac{\partial f+\varepsilon f^{*}}{\partial x_{2}}, \frac{\partial f+\varepsilon f^{*}}{\partial x_{1}}\right)\left.\right|_{p}> \\
& =<\mathbf{a}+\varepsilon \mathbf{a}^{*},\left(\frac{\partial f}{\partial x_{1}}+\varepsilon \frac{\partial f^{*}}{\partial x_{1}}, \frac{\partial f}{\partial x_{2}}+\varepsilon \frac{\partial f^{*}}{\partial x_{2}}, \frac{\partial f}{\partial x_{3}}+\varepsilon \frac{\partial f^{*}}{\partial x_{3}}\right)> \\
& =<\mathbf{a}+\varepsilon \mathbf{a}^{*},\left(\frac{\partial f}{\partial x_{1}}, \frac{\partial f}{\partial x_{2}}, \frac{\partial f}{\partial x_{3}}\right)+\varepsilon\left(\frac{\partial f^{*}}{\partial x_{1}}, \frac{\partial f^{*}}{\partial x_{2}}, \frac{\partial f^{*}}{\partial x_{3}}\right)> \\
& =<\mathbf{a}+\varepsilon \mathbf{a}^{*}, \overrightarrow{\nabla f}+\varepsilon \overrightarrow{\nabla f^{*}}> \\
& =<\mathbf{a}+\varepsilon \mathbf{a}^{*}, \overrightarrow{\nabla f}>+\varepsilon\left[<\mathbf{a}^{*}, \overrightarrow{\nabla f}>+<\overrightarrow{\nabla f^{*}}, \mathbf{a}>\right] .
\end{aligned}
$$

Theorem 6. Let the $\forall F, G: \mathbb{D}^{n} \longrightarrow \mathbb{D}$ be two differentiable function if $\vec{A}_{p}$ and $\vec{B}_{p} \in \mathbb{D}^{n}$ dual vectors and $\forall c_{1}, c_{2} \in \mathbb{R}$;

(i) $\left(c_{1} \vec{A}_{p}+c_{2} \vec{B}_{p}\right)[F]=c_{1} \vec{A}_{p}[F]+c_{2} \vec{B}_{p}[F]$

(ii) $\vec{A}_{p}\left[c_{1} F+c_{2} G\right]=c_{1} \vec{A}_{p}[F]+c_{2} \vec{A}_{p}[G]$

(iii) $\vec{A}_{p}[F . G]=\vec{A}_{p}[F] G(P)+F(P) \vec{A}_{p}[G]$

are obtained.

Proof.

(i)

$$
\begin{aligned}
\left(c_{1} \vec{A}_{p}+c_{2} \vec{B}_{p}\right)[F] & =<\left(c_{1} \vec{A}_{p}+c_{2} \vec{B}_{p}\right),\left.\overrightarrow{\nabla F}\right|_{p}> \\
& =<c_{1} \vec{A}_{p},\left.\overrightarrow{\nabla F}\right|_{p}>+<c_{2} \vec{B}_{p},\left.\overrightarrow{\nabla F}\right|_{p}> \\
& =c_{1}<\vec{A}_{p},\left.\overrightarrow{\nabla F}\right|_{p}>+c_{2}<\vec{B}_{p},\left.\overrightarrow{\nabla F}\right|_{p}> \\
& =c_{1} \vec{A}_{p}[F]+c_{2} \vec{B}_{p}[F] .
\end{aligned}
$$

(ii)

$$
\begin{aligned}
\vec{A}_{p}\left[c_{1} F+c_{2} G\right] & =<\vec{A}_{p}, \overrightarrow{\nabla\left(c_{1} F+c_{2} G\right)}> \\
& =<\vec{A}_{p}, \overrightarrow{\nabla\left(c_{1} F\right)}+\overrightarrow{\nabla\left(c_{2} G\right)}> \\
& =<\vec{A}_{p}, c_{1} \overrightarrow{\nabla F}+c_{2} \overrightarrow{\nabla G}> \\
& =<\vec{A}_{p}, c_{1} \overrightarrow{\nabla F}>+<\vec{A}_{p}, c_{2} \overrightarrow{\nabla G}> \\
& =c_{1}<\vec{A}_{p}, \overrightarrow{\nabla F}>+c_{2}<\vec{A}_{p}, \overrightarrow{\nabla G}> \\
& =c_{1} \vec{A}_{p}[F]+c_{2} \vec{A}_{p}[G] .
\end{aligned}
$$


(iii)

$$
\begin{aligned}
\mathbf{A}_{p}[F \cdot G] & =<\mathbf{A}_{p},\left.\overrightarrow{\nabla(F \cdot G)}\right|_{p}> \\
& =<A_{p}, \overrightarrow{\nabla F} \cdot G(p)+F(p) \cdot \overrightarrow{\nabla G}> \\
& =<A_{p}, \overrightarrow{\nabla F} \cdot G(p)>+<A_{p}, F(p) \cdot \overrightarrow{\nabla G}> \\
& =<A_{p}, \overrightarrow{\nabla F}>\cdot G(p)+<A_{p}, \overrightarrow{\nabla G}>\cdot F(p) \\
& =A_{p}[F] \cdot G(p)+A_{p}[G] \cdot F(p) .
\end{aligned}
$$

Definition 5. For the $\forall P \in \mathbb{D}^{n}$ dual point,

$$
\begin{array}{r}
\chi: \mathbb{D}^{n} \rightarrow \bigcup_{P \in \mathbb{D}^{n}} T_{\mathbb{D}^{n}}(P) \\
p \rightarrow \mathbf{A}_{\mathbf{p}}
\end{array}
$$

transformation at $\mathbb{D}^{n}$ is called the dual vector field. Besides,

$$
\chi\left(\mathbb{D}^{n}\right)=\left\{\mathbf{A} \mid \mathbf{A}: \mathbb{D}^{n} \rightarrow \bigcup \mathbb{T}_{\mathbb{D}^{n}}(P)\right\}
$$

set at $\mathbb{D}^{n}$ is called as the set of dual vector fields. For the $\forall \mathbf{A}, \mathbf{B} \in \chi\left(\mathbb{D}^{n}\right)$ dual vector fields and for the $\forall P \in \mathbb{D}_{n}$ dual point,

$$
\begin{array}{r}
(\mathbf{A} \oplus \mathbf{B})(p)=\mathbf{A}_{p} \oplus \mathbf{B}_{p} \\
(c \odot \mathbf{A})(P)=c \odot \mathbf{A}_{p}
\end{array}
$$

$\left\{\chi\left(\mathbb{D}^{n}\right), \oplus, \mathbb{R},+, ., \odot\right\}$ vector space is called as the space of dual vector fields.

Definition 6. F be differentiable dual function for $\forall P \in \mathbb{R}^{n}$, the $\vec{A} \in \chi\left(\mathbb{D}^{n}\right)$, which is defined with $F$ be differentiable dual function for

$$
A[F](P)=A_{p}[F]
$$

is called $A[F] \in C\left(\mathbb{D}^{n}, \mathbb{D}\right)$ the derivation of $F$ dual function in the direction of the dual vector field $A$.

Theorem 7. $\forall \vec{A}, \vec{B} \in \chi\left(\mathbb{D}^{n}\right)$ dual vector fields and $\forall F, G, H \in C\left(\mathbb{D}^{n}, \mathbb{D}\right)$ dual functions are given. For $\forall c_{1}, c_{2} \in \mathbb{R}$,

(i) $\quad(F . A+G . B) \cdot[H]=F \cdot A[H]+G \cdot B[H]$

(ii) $A\left[c_{1} \cdot F+c_{2} \cdot G\right]=c_{1} \cdot A[F]+c_{2} \cdot A[G]$

(iii) $A[F \cdot G]=A[F] \cdot G+F \cdot A[G]$

properties are obtained.

Proof. For $f, f^{*}, g, g^{*}, h, h^{*} \in C\left(E^{n}, \mathbb{R}\right)$ real functions, the dual functions $F=f+\varepsilon f^{*}, G=g+\varepsilon g^{*}, H=h+\varepsilon h^{*}$ are given. The dual vector $\mathbf{A}=\mathbf{a}+\varepsilon \mathbf{a}^{*}$ is given for $\vec{a}=\left(a_{1}, a_{2}, a_{3}\right), \vec{a}^{*}=\left(a_{1}^{*}, a_{2}^{*}, a_{3}^{*}\right)$ real vectors. By benefiting from the definition (6); 
(i)

$$
\begin{aligned}
(F \cdot A+G . B)[H](P) & =(F \cdot A+G \cdot B)_{p}[H] \\
& =\left((F \cdot A)_{p}+(G \cdot B)_{p}\right)[H] \\
& =(F \cdot A)_{p}[H]+(G B)_{p}[H] \\
& =F(P) \cdot A_{p}[H]+G(P) \cdot B_{p}[H] \\
& =F(P) \cdot A[H](P)+G(P) \cdot B[H](P) \\
& =F(P) \cdot A[H]+G(P) \cdot B[H]
\end{aligned}
$$

(ii)

$$
\begin{aligned}
A\left[c_{1} F+c_{2} G\right](P) & =A_{p}\left[c_{1} F+c_{2} G\right] \\
& =A_{p}\left[c_{1} F\right]+A_{p}\left[c_{2} G\right] \\
& =c_{1} A_{p}[F]+c_{2} A_{p}[G] \\
& =c_{1} A[F](P)+c_{2} A[G](P)
\end{aligned}
$$

(iii)

$$
\begin{aligned}
A[F \cdot G](P) & =A_{p}[F \cdot G] \\
& =A_{p}[F] \cdot G(P)+F(P) \cdot A_{p}[G] \\
& =A[F](P) \cdot G(P)+F(P) \cdot A[G](P)
\end{aligned}
$$

the above equations are obtained.

\section{Numerical example}

After finding the unit dual vector $\mathbf{A}=\mathbf{a}+\varepsilon \mathbf{a}^{*}$ which corresponds the line in $\vec{a}=\left(\frac{2}{3}, \frac{2}{3}, \frac{1}{3}\right)$ vector direction, which passes through $M(1,2,2)$ points, let us compute the derivation in the direction of $\mathbf{A}$ unit dual vector of the dual function $\mathbf{F}=\mathbf{f}+\varepsilon \mathbf{f}^{*}$ defined by $f=x_{1} \cdot x_{2}+2 \cdot x_{3}$ and $f^{*}=x_{1} \cdot x_{2}-2 x_{3}$ real vectors at the $P(1,2,2,0,0,0) \in \mathbb{D}^{3}$ point.

Firstly, let us find the line equation to which the dual vector given in the example corresponds. As it is given in the Figure 1 , the line equation is found via:

$$
\overrightarrow{O X}=\overrightarrow{O M}+\lambda \mathbf{a}
$$

In the equation (3), $X(x, y, z), M(1,2,2)$ and $\vec{d}=\left(\frac{2}{3}, \frac{2}{3}, \frac{1}{3}\right)$ vectors place them and solve the equation,

$$
\begin{aligned}
(x, y, z) & =\left(1+\lambda \frac{2}{3}, 2+\lambda \frac{2}{3}, 2+\lambda \frac{1}{3}\right) \\
\lambda & =\frac{x-1}{2 / 3}=\frac{y-2}{2 / 3}=\frac{z-2}{1 / 3} \\
\frac{x-1}{2} & =\frac{y-2}{2}=\frac{z-2}{1}
\end{aligned}
$$


We will have the above line equation. In order to find the dual vector which corresponds to this directed line, we must find the a moment which is vertical to the a vector. It is observed that the moment is:

$$
\mathbf{a}^{*}=\mathbf{O M} \wedge \mathbf{a}=\left|\begin{array}{lll}
\mathbf{i} & \mathbf{j} & \mathbf{k} \\
1 & 2 & 2 \\
\frac{2}{3} & \frac{2}{3} & \frac{1}{3}
\end{array}\right|=\left(-\frac{2}{3}, 1,-\frac{2}{3}\right)
$$

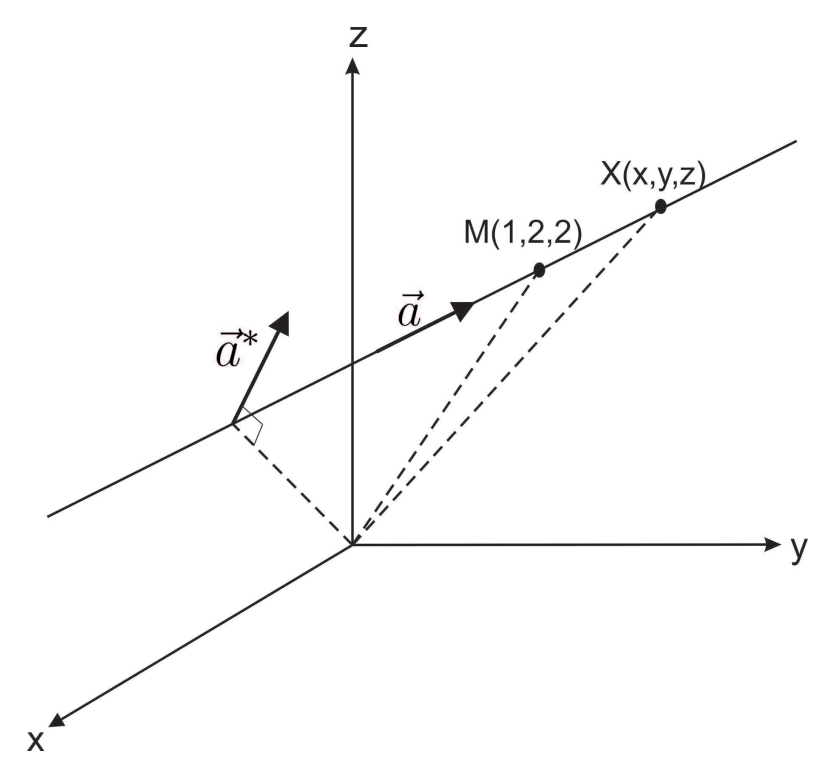

Fig. 1: The directed line to which the unit dual vector corresponds.

Now, let us find the derivation of the dual function $F=\left(x_{1} \cdot x_{2}+2 x_{3}\right)+\varepsilon\left(x_{1} \cdot x_{2}-2 x_{3}\right)$ in the direction of dual vector $\mathbf{A}_{p}=\left.\left(\mathbf{a}+\varepsilon \mathbf{a}^{*}\right)\right|_{p}$. Therefore, we write

$$
\begin{aligned}
A_{p}[F] & =<\mathbf{A}_{p},\left.\overrightarrow{\nabla F}\right|_{p}> \\
& =<\left.\left(\mathbf{a}+\varepsilon \mathbf{a}^{*}\right)\right|_{p}, \overrightarrow{\nabla f}+\varepsilon \overrightarrow{\nabla f^{*}}> \\
& =<\left(\frac{2}{3}, \frac{2}{3}, \frac{1}{3}\right)+\varepsilon\left(-\frac{2}{3}, 1,-\frac{2}{3}\right),\left(x_{2}, x_{1}, 2\right)+\varepsilon\left(x_{2}, x_{1},-2\right)> \\
& =<\left(\frac{2}{3}-\varepsilon \frac{2}{3}, \frac{2}{3}+\varepsilon, \frac{1}{3}-\varepsilon \frac{2}{3}\right),\left(x_{2}+\varepsilon x_{2}, x_{1}+\varepsilon x_{1}, 2-2 \varepsilon\right)>\left.\right|_{p} \\
& =<\left(\frac{2}{3}-\varepsilon \frac{2}{3}, \frac{2}{3}+\varepsilon, \frac{1}{3}-\varepsilon \frac{2}{3}\right),(2+\varepsilon 2,1+\varepsilon, 2-2 \varepsilon)> \\
& =\frac{4}{3}-\frac{4}{3} \varepsilon+\frac{4}{3} \varepsilon+\frac{2}{3}+\varepsilon+\frac{2}{3} \varepsilon+\frac{2}{3}-\varepsilon \cdot \frac{4}{3}-\frac{2}{3} \varepsilon \\
& =\left(\frac{4}{3}+\frac{2}{3}+\frac{2}{3}\right)+\varepsilon\left(-\frac{4}{3}+\frac{4}{3}-\frac{4}{3}+1\right) \\
& =\frac{8}{3}-\varepsilon \frac{1}{3} \in \mathbb{D} .
\end{aligned}
$$




\section{Conclusion}

In this study, the subject of directional derivative, which is one of the most important subjects of differential geometry, has been studied for dual spaces. By making use of the knowledge which says that a dual unit vector corresponds to a directed line in the Euclidean Space, and with the help of the E. Study conversion in dual spaces, the unit dual vector, to which a line whose direction and one point on it corresponds, has been found and drawn. Then, the derivation of a dual function in the direction of unit dual vector has been computed. It is considered that this study will be a pioneer for some future studies on the screw theory and dual space.

\section{Competing interests}

The authors declare that they have no competing interests.

\section{Authors' contributions}

All authors have contributed to all parts of the article. All authors read and approved the final manuscript.

\section{Acknowledgements}

The authors wish to thank the referees for their careful corrections and extensive comments.

\section{References}

[1] Kandasamy, W.B.V., and Smarandache, F., Dual Numbers, Zip Publishing, Ohio, 2012.

[2] Study, E.,Die Geometrie der Dynamen, Leibzig, 1903.

[3] Kazaz, M., Ozdemir, A., Ugurlu H.H.,Eliptic Motion on Dual Hyperbolic Unit Sphere $\widetilde{H}_{0}^{2}$, Mechanism Machine Theory, 14501459, 2009.

[4] Veldkamp, G. R., On the Use of Dual Numbers, Vectors and Matrices in Instantaneous Spatial Kinematics, Mechanisms and Machine Theory, vol. 11 pp. 141-156, 1976.

[5] Taleshian, A., Application of Covariant Derivative in the Dual Space, Int. J. Contemp. Math. Sciences, Vol. 4, no. 17, 821-826, 2009.

[6] Ball, R.S., Theory of Screws, Cambridge University Press, Cambridge, 1900.

[7] O’Neill, B., Elementary Geometry Differential, New York and London, 1966.

[8] Do Carmo, M.P., Differential Geometry Curves and Surfaces, Prentice Hall, Englewood Cliffs, 1976. 